\title{
A single-center descriptive study of untraced sources of infection among new cases of coronavirus disease in Tokyo, Japan
}

\author{
Sayaka Hikida', Shinichiro Morioka ${ }^{1,2,3, *}$, Naoki Fujii ${ }^{3}$, Taichi Tajima ${ }^{3}$, Yuriko Terayama ${ }^{4}$, Yuriko Sugiura ${ }^{4}$, Masahiro \\ Ishikane ${ }^{1,3}$, Jin Takasaki ${ }^{4}$, Masayuki Hojo ${ }^{4}$, Norio Ohmagari ${ }^{1,3}$ \\ ${ }^{1}$ Disease Control and Prevention Center, National Center for Global Health and Medicine Hospital, Tokyo, Japan; \\ ${ }^{2}$ Emerging and Re-emerging Infectious Diseases, Graduate School of Medicine, Tohoku University, Sendai, Japan; \\ ${ }^{3}$ AMR Clinical Reference Center, National Center for Global Health and Medicine Hospital, Tokyo, Japan; \\ ${ }^{4}$ Division of Respiratory Medicine, National Center for Global Health and Medicine Hospital, Tokyo, Japan.
}

\begin{abstract}
We investigated possible sources of newly infected patients with coronavirus disease (COVID-19) after the fourth wave in order to explore unknown sources. Retrospective chart review on all the confirmed patients with COVID-19 admitted to the National Center for Global Health and Medicine (NCGM) in Tokyo, Japan was conducted from May 22 through June 29, 2021. Among the 22 participants, 14 (64\%) had a history of known highrisk infection behaviors. Of those, 12 reported that their activities involved eating and drinking. In addition, there were 24 high-risk situations, of those, 21 (88\%) were related to indoor dining, and masks were not worn in 22 situations (92\%). New source of infection has not been identified. In situations with a high known risk of infection, many cases were related to eating and drinking, and insufficient use of masks was evident. Raising risk awareness on infection prevention and control of COVID-19 is urgently needed.
\end{abstract}

Keywords: coronavirus disease, untraced sources of infection, high-risk infection behavior, indoor dining, mask

\section{Introduction}

Since the end of 2019, an accumulation of severe pneumonias of unknown cause was reported in Wuhan City, Hubei Province, China, which was subsequently found to be a coronavirus disease (COVID-19) (1). In Japan, there have been four waves of COVID-19 as of August 1, 2021 (2). Even if the number of new cases declines, there may be an unknown source of infection, which may serve as the origin of the next wave of severe acute respiratory syndrome coronavirus 2 (SARS-CoV-2) infection. When the number of newly infected patients in the fourth wave showed a downward trajectory, we conducted an exploratory investigation into possible sources of infection of newly infected patients after the fourth wave.

A review on confirmed patients with COVID-19 conducted after the fourth wave

We conducted a retrospective chart review on all the confirmed patients with COVID-19 admitted to the National Center for Global Health and Medicine (NCGM) in Tokyo, Japan, from May 22 through June 29, 2021. As part of their daily patient care, we conducted oneon-one individual interviews with inpatients with
COVID-19. Each interview lasted approximately 20 minutes. The participants were COVID-19 patients aged 20 years or older who were admitted to the NCGM from May 22 to June 29, 2021, excluding those who had a clear source of infection (e.g., close contact or cluster infection) at the time of admission and those with whom it was difficult to communicate. They were asked to fill out a behavioral questionnaire (Appendix 1, https:// www.globalhealthmedicine.com/site/supplementaldata. html?ID=26) (3) during hospitalization, and based on the questionnaire, interviews were conducted by four researchers (SH, SM, NF, TT) based on an interview guide (Appendix 2, https://www.globalhealthmedicine. com/site/supplementaldata.html?ID=26) from June 10 to July 1, 2021. The researchers were two infectious disease physicians and two infection control nurses. The physicians were involved in COVID-19 patient care, while the nurses were not.

The following items were reviewed: patient demographics (age, sex, nationality); whether COCOA (a contact tracing app) was installed; if installed, whether COCOA notification was given; behavioral history/ contact history from 14 days to 1 day before the onset of illness and the location and date of the contact; mask-wearing behavior; and the contact situation in detail. Participants' behavioral history/contact history 
was classified into two categories: known high-risk situations and no known high-risk situations. Known high-risk situations included travel by ferryboats or long-distance buses; attending gyms, indoor music events, nightclubs, standing parties, karaoke, indoor dining, or other types of poorly ventilated, enclosed indoor gatherings; and a history of staying in endemic areas (either domestic or international) (3). Particular attention was paid to situations in which face-masks were removed, particularly during the 4 to 7 days before the onset, which is the most likely window of exposure based on the incubation period (4). Next, the number of participants who were in situations with a known high risk of infection was also tabulated by the situation. We then conducted a qualitative analysis of the information given during the interview regarding patient's thoughts and beliefs about the possible sources of infection. The study protocol was reviewed and approved by the Ethics Committee of the Center Hospital of the NCGM after compliance with the condition that a document that declares an opt-out policy by which any potential participant and/or their relatives could refuse to be included in this study was uploaded on the website of the Center Hospital of the NCGM.

Among the 43 COVID-19 patients aged 20 years or older who were admitted to the NCGM Hospital during the study period, 29 were included, excluding nine patients with a clear source of infection at the time of admission, three patients on a ventilator, one patient who was difficult to interview because of his general condition, and one patient who was difficult to interview because of a language barrier. Among the 29 patients, 23 were interviewed. Three patients were discharged from the hospital prior to the interview, and three were not interviewed immediately after admission. Among the 23 participants, one incorrectly stated the date of onset as the date of hospitalization, and the interview was therefore considered invalid. The responses of the 22 participants were analyzed.

\section{The risk of infection based on the behavioral/contact histories}

Among the 22 participants, 17 (77\%) were male and five $(23 \%)$ were female; the median age was 52.5 years (interquartile range: 44-66 years), and 19 (86\%) were Japanese. Six of the 22 participants $(27 \%)$ had downloaded the COCOA notification app, none of whom had received a notification. The behavioral/ contact histories of the participants were categorized into situations with known high risk of infection and the others, as shown in Table 1. We classified the behavioral/ contact histories into two categories: We categorized them into two groups: those with a high risk of infection $(n=24)$, including indoor dining $(n=21)$, attending an indoor music event $(n=1)$, or attending gym $(n=2)$, and those with a low risk of infection $(n=118)$, including shopping $(n=34)$, working $(n=22)$, using public transportation $(n=31)$, visiting medical institutions $(n=$ $14)$, leisure activities $(n=6)$, and others $(n=11)$. Among the 24 high-risk situations, $21(88 \%)$ were related to indoor dining, and masks were not worn in 22 situations $(92 \%)$.

With regard to the participants who were in known high-risk situations, 12 out of 22 (55\%) reported that their activities involved eating or drinking. Of these, six reported having dinner with multiple people, including friends, and of these, five had dinner at a restaurant, three at a pub or a bar, one at a birthday party with more than 20 people, and one at a dinner party using a delivery service (with some participants reporting several of these activities). In addition, one person attended an indoor music concert without wearing a mask, one person used a gym, and one person was a gym instructor. There was one person overlapping with the 12 participants whose activities involved eating and drinking.

Examples of thoughts and beliefs of the patients who thought they could contribute to infection, include: "I don't think that I need to wear a mask while traveling except by train or at work"; "I think that it is okay to eat while chatting in the company cafeteria"; "I think that it is okay for staff to talk to each other without masks after work"; "I feel that it is not good, but I don't want to wear a mask at a concert venue"; "I didn't know that eating out was a risk for infection"; "It was a private barbershop, so there was no need to wear a mask"; and "If it was a private golf game, there would be no need to wear a mask even if there were four of us".

\section{Enhancing risk awareness and promoting infection prevention}

One of the most important findings in this study was that masks were not worn in $22(92 \%)$ of the 24 known high-risk infection situations. In addition, 14 of the 22 participants $(64 \%)$ had a history of known high-risk infection behaviors. With regard to patients' thoughts and beliefs that could contribute to infection, the participants had opportunities to become infected in well-known high-risk situations. This suggests that participants had a low level of awareness of infection control or a lack of knowledge about infection control. Since this survey was conducted after the fourth wave, decreased interest in infection risk information, decreased concern, and optimism bias (5) may have contributed the infections. There is a concern about the spread of COVID-19 variant (6), it is necessary to consider effective countermeasures for providing accurate information and fostering risk awareness so that they can lead to infection prevention measures.

The second important finding was that there were situations where three participants were teaching karaoke, met a friend who tested positive, and had a oneon-one lesson with a gym instructor with their masks 
Table 1. Possible sources of exposure based on behavioral histories from 14 days to 1 day before the onset

\begin{tabular}{|c|c|c|}
\hline Activity type & Activity & $n$ \\
\hline \multicolumn{3}{|l|}{ Known high risk } \\
\hline \multirow[t]{15}{*}{ Indoor dining } & & 21 \\
\hline & Eating out with friends & 3 \\
\hline & $\underline{\underline{\text { Ramen shop }}}$ & 3 \\
\hline & Soba shop & 2 \\
\hline & Izakaya with friends & 2 \\
\hline & Chinese restaurant & 2 \\
\hline & $\overline{\text { Chinese restaurant }}$ & 1 \\
\hline & Izakava with friends & 1 \\
\hline & A birthday party at a pub with close contact with about 30 people & 1 \\
\hline & Japanese restaurant & 1 \\
\hline & Company cafeteria & 1 \\
\hline & Eating out with friends & 1 \\
\hline & Eating out alone & 1 \\
\hline & $\overline{\text { Eating out alone }}$ & 1 \\
\hline & Order a home delivery service and have a meal with 4 people & 1 \\
\hline \multirow{2}{*}{ Indoor music events } & & 1 \\
\hline & Attend a piano concert with vocalists & 1 \\
\hline \multirow[t]{3}{*}{ Gyms } & & 2 \\
\hline & Use of fitness club & 1 \\
\hline & Instructor & 1 \\
\hline \multirow{13}{*}{ Shopping } & \multicolumn{2}{|c|}{ Others } \\
\hline & Shonnino at sunermarkets & $\begin{array}{r}34 \\
8\end{array}$ \\
\hline & Convenience store & 8 \\
\hline & Shopping at supermarkets & 5 \\
\hline & Convenience store & 3 \\
\hline & Shopping at department stores & 2 \\
\hline & Florist & 2 \\
\hline & Convenience store & 1 \\
\hline & Convenience store & 1 \\
\hline & Shopping at department stores & 1 \\
\hline & Real estate agent & 1 \\
\hline & Pharmacy & 1 \\
\hline & Bicycle shop & 1 \\
\hline \multirow[t]{11}{*}{ Workplace/work-related } & & 22 \\
\hline & Meetings and interviews at the workplace & 10 \\
\hline & 1-2-hour conversation with colleague after work - colleague tested positive & 3 \\
\hline & Meetings and interviews at the workplace & 2 \\
\hline & Teaching karaoke & 1 \\
\hline & One-on-one lessons as an instructor & 1 \\
\hline & Office work at a facility for the disabled & 1 \\
\hline & Cleaning the bullet train & 1 \\
\hline & Transportation of users of the facility & 1 \\
\hline & Serving customers at cafe & 1 \\
\hline & Brushing teeth in the bathroom at work & 1 \\
\hline \multirow[t]{11}{*}{ Public transportation and transportation-related } & & 31 \\
\hline & Bicycling & 8 \\
\hline & Commute by train & 5 \\
\hline & Bicycling & 4 \\
\hline & Commute by foot & 4 \\
\hline & Commute by train & 3 \\
\hline & Commute by foot & 2 \\
\hline & Commute to work & 2 \\
\hline & Commute by train & 1 \\
\hline & Commute by foot & 1 \\
\hline & $\overline{\text { Commute by cab }}$ & 1 \\
\hline Medical institution & & 14 \\
\hline & Clinic & 5 \\
\hline & Vaccination site & 3 \\
\hline & Hospital & 2 \\
\hline & Hospital & 1 \\
\hline & Clinic & 1 \\
\hline & Dentist & 1 \\
\hline & $\overline{\text { Dentist }}$ & 1 \\
\hline Leisure & & 6 \\
\hline & A walk & 3 \\
\hline & Golf with family and friends & 2 \\
\hline & A walk in the red-light district & 1 \\
\hline Others & & 11 \\
\hline & Use of delivery service & 2 \\
\hline & Bank & 2 \\
\hline & Bank & 1 \\
\hline & Meeting with a friend who tested positive & 1 \\
\hline & Hotel (cleaning staff's work was messy) & 1 \\
\hline & Barbershop & 1 \\
\hline & Barbershop & 1 \\
\hline & Visit of the glass replacement company & 1 \\
\hline & $\underline{\text { Visit to relative's house }}$ & 1 \\
\hline
\end{tabular}

Places where the mask was removed are underlined. Activities that took place 4 to 7 days before the onset are in bold. 
on. Even if a mask is worn, it is not $100 \%$ effective at preventing infection (7).

The third important finding was that there were situations in which two participants did not wear a mask during dental procedures and in barber shops. It is possible that the risk of infection was increased due to the lack of mask use, but it is unclear whether the participants actually became infected through these activities. In this study, we were not able to identify any new source of infection that had not been previously identified.

This study had several limitations. First, we examined the relationship between the participants and interviewers. Two of the interviewers were physicians in the department of infectious diseases who had treated some of the participants, and this may have affected their responses during the interview. Second, there was recall bias. Third, there is a possibility that some of the participants intentionally did not report situations in which there was a known high risk of infection. Fourth, only one interview was conducted with each participant, and the content of the participants' answers was not reconfirmed. Fifth, this study was not a prospective qualitative study, but a retrospective chart analysis. Thus, the sample size was small and the qualitative information might not be saturated. Sixth, it is not known whether participants wore masks appropriately, because this was not verified. Last, the percentage of infections caused by the Delta variant ranged from $1.5 \%$ to $31.6 \%$ during the survey period ( 8 ), and the survey was conducted at a time when the proportion of infections due to the Delta variant was relatively lower than during the fifth wave.

The urgency of providing accurate information and raising risk awareness on infection prevention and control of COVID-19 in the absence of identified new sources of infection

When the number of newly infected persons in the fourth wave showed a decreasing trend, we investigated the sources of infection of newly infected persons. In situations with a high known risk of infection, many of the cases were related to eating and drinking, and we did not identify any new sources of infection. In situations with a high known risk of infection, insufficient use of masks was evident, and there was a low level of awareness and lack of knowledge about infection control. Providing accurate information and raising risk awareness on infection prevention and control of COVID-19 is urgently needed.

\section{Funding: None.}

Conflict of Interest: The authors have no conflicts of interest to disclose.

\section{References}

1. Hayakawa K, Kutsuna S, Kawamata T, et al. SARS$\mathrm{CoV}-2$ infection among returnees on charter flights to Japan from Hubei, China: a report from National Center for Global Health and Medicine. Glob Health Med. 2020; 2:107-111.

2. Ministry of Health, Labour and Welfare, Japan. Visualizing the data: information on COVID-19 infections. https:// covid19.mhlw.go.jp/en/ (accessed August 2, 2021).

3. New Coronavirus Infection Patient Behavior Survey Form (Source of Infection). https://www.fukushihoken.metro. tokyo.lg.jp/nisitama/topics/covid19/yousei.files/chousa2. $p d f$ (accessed August 2, 2021). (in Japanese)

4. Li Q, Guan X, Wu P, et al. Early Transmission dynamics in Wuhan, China, of novel coronavirus-infected pneumonia. N Engl J Med. 2020; 382:1199-1207.

5. Oikawa H, Oikawa M. Cognitive, affective and behavioral changes in crisis: preventing swine flu infection. Shinrigaku Kenkyu. 2010; 81:420-425. (in Japanese).

6. Cascella M, Rajnik M, Aleem A, Dulebohn SC, Di Napoli R. Features, Evaluation, and Treatment of Coronavirus (COVID-19). 2021 Jul 17. In: StatPearls [Internet]. Treasure Island (FL): StatPearls Publishing; 2021.

7. Ueki H, Furusawa Y, Iwatsuki-Horimoto K, Imai M, Kabata H, Nishimura H, Kawaoka Y. Effectiveness of face masks in preventing airborne transmission of SARSCoV-2. mSphere. 2020; 5:e0637-20.

8. Screening results at the Tokyo Metropolitan Health and Safety Research Center. https://www.fukushihoken.metro. tokyo.lg.jp/iryo/kansen/corona_portal/henikabu/screening. files/screening_072902.pdf(accessed August 2, 2021).

Received August 5, 2021; Accepted August 9, 2021.

Released online in J-STAGE as advance publication August 10, 2021.

*Address correspondence to:

Shinichiro Morioka, Disease Control and Prevention Center, National Center for Global Health and Medicine Hospital. 1-21-1 Toyama, Shinjuku-ku, Tokyo 162-8655, Japan.

E-mail: shmorioka@hosp.ncgm.go.jp 